\title{
A swollen wrist
}

\author{
Dzmitry Fursevich $^{1}$ - David Warden ${ }^{1} \cdot$ Christopher Wasyliw $^{1} \cdot$ Laura Bancroft $^{1}$. \\ Kurt Scherer ${ }^{1}$
}

Published online: 11 January 2016

(C) ISS 2016

\section{Discussion}

The diagnosis of mycobacterial tenosynovitis was suggested based on the imaging and clinical findings (Fig. 1 ), and the patient had a radical tenosynovectomy less than a month after presentation. During the surgical intervention, numerous rice bodies were extracted from the tendon sheath and bursae. The wrist joint was not involved. Culture of the surgical specimen was positive for Mycobacterium tuberculosis.

Mycobacterium tuberculosis infection of the musculoskeletal system accounts for up to $25 \%$ of all extrapulmonary cases of tuberculosis [1]. Infection of the tendon sheaths and synovial bursae of the wrist and hand is exceedingly rare, and may be difficult to diagnose prospectively, especially in cases without any prior history of tuberculosis exposure [2]. Because radial and ulnar bursae frequently communicate with each other and with flexor tendon sheaths [3, 4], the infection tends to spread in a predictable pattern resulting in an "hourglass" or "horseshoe" configuration of the distended synovial structures. The presenting symptoms are often

The case presentation can be found at doi: 10.1007/s00256-015-2322-2

Dzmitry Fursevich

dzmitry.fursevich.md@flhosp.org

1 Department of Diagnostic Radiology, Florida Hospital, 601 E Rollins St, Orlando, FL 32803, USA nonspecific and include wrist pain, swelling, and loss of range of motion. Laboratory tests are usually not helpful, although occasionally elevated erythrocyte sedimentation rate and Creactive protein levels may be detected [5]. Delay from the onset of symptoms to an accurate diagnosis may range from 3 to 24 months. If not detected early, tuberculous tenosynovitis of the wrist can lead to complications of carpal tunnel syndrome, tendon disruption and osteomyelitis [6].

Both ultrasound and magnetic resonance imaging (MRI) can be very helpful in evaluation of suspected wrist tenosynovitis. The unique nature of rice bodies in tuberculous tenosynovitis can be identified on MRI and help distinguish it from other conditions such as extra-articular synovial chondromatosis and pigmented villonodular tenosynovitis (PVNS). Rice bodies are the hallmark of tuberculous infection of the wrist, and were present in 7 out of 8 cases in one case series [6]. Because they are composed of fibrin, rice bodies are generally hypointense on T1- and T2-weighted images. In contrast, non-calcified lesions of extraarticular synovial chondromatosis are cartilaginous in nature and may be slightly hyperintense on T1- and T2-weighted images [7]. Calcifications of synovial osteochondromatosis can be excluded by radiography or computed tomography (CT). Nodules of PVNS contain hemosiderin and can be differentiated from the rice bodies utilizing gradient echo MRI sequences, where hemosiderin blooming will occur [8]. It is important to note that MRI cannot distinguish tuberculous tenosynovitis from either atypical mycobacterial tenosynovitis (Fig. 2) or rheumatoid tenosynovitis, both of which can present with rice body formation and synovial inflammation [5]. History of penetrating injury or elevated rheumatoid factor can be helpful in those cases. 
Fig. 1 a Non-contrast axial T1weighted sequence and $\mathbf{b}$-d fat suppressed axial, coronal and sagittal T2-weighted sequences demonstrate numerous T1- and T2-hypointense lesions expanding the flexordigitorum tendon sheaths and involving the palmar, radial and ulnar bursae. The lesions are contained bythe bursal synovium resulting in the hourglass configuration of the bursae on the coronal images

Fig. 2 Mycobacterium avium tenosynovitis in a 77-year-old man. a Non-contrast coronal T1weighted and $\mathbf{b}$ post-contrast fatsuppressed coronal T1-weighted sequences demonstrate marked distention of the flexor pollicis longus tendon with synovial thickening and enhancement. c-d Fat suppressed T2-weighted coronal and sagittal sequences demonstrate numerous rice bodies within the tendon sheath and radial bursa. Surgical specimen was positive for Mycobacterium avium complex
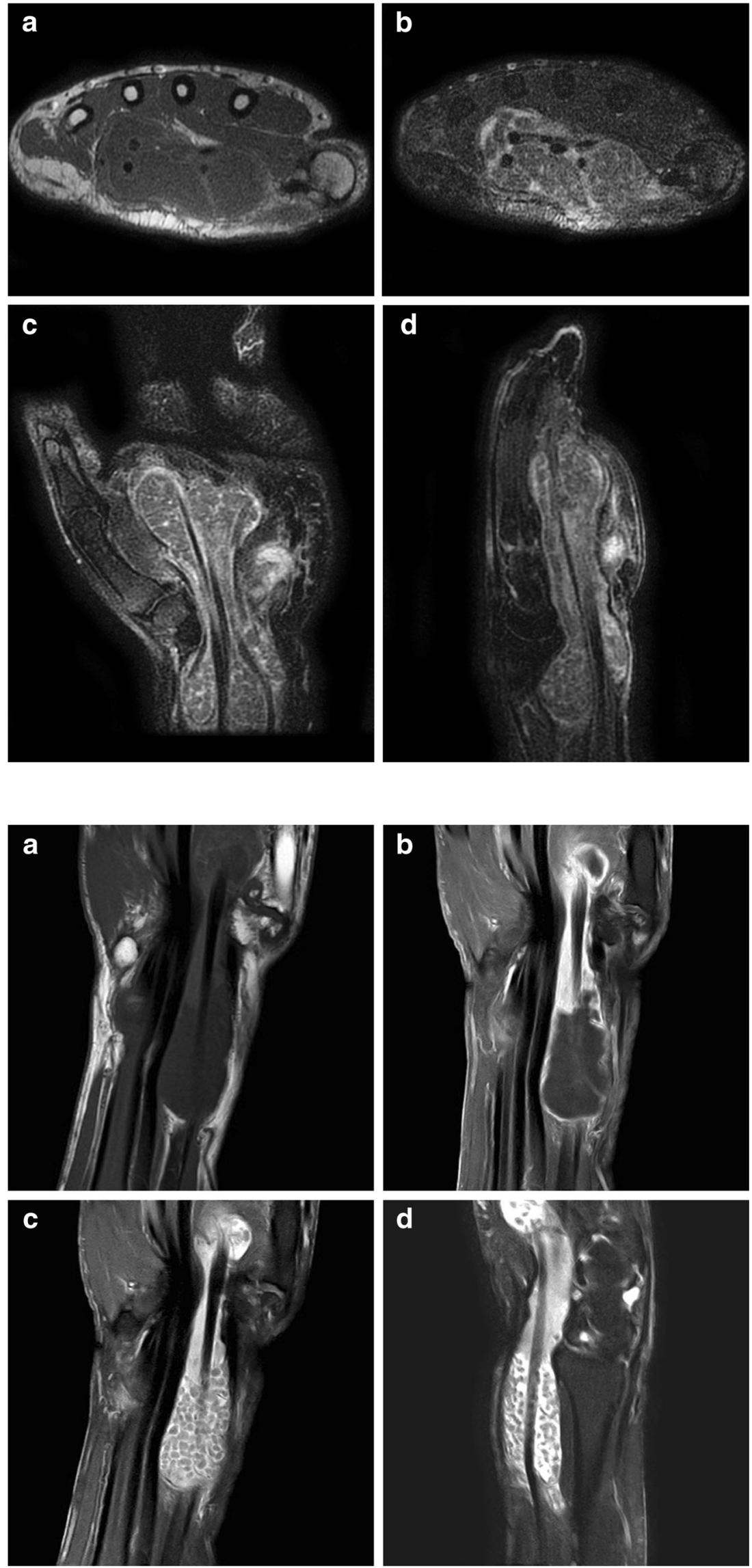
In summary, tuberculous tenosynovitis of the hand and wrist should always be suspected in cases when rice bodies are detected on MR imaging together with thickened, enhancing synovium. Differential diagnosis includes PVNS, synovial chondromatosis and osteochondromatosis, rheumatoid tenosynovitis and atypical mycobacterial tenosynovitis, with the last two entities indistinguishable from tuberculous tenosynovitis on MRI. Early diagnosis is essential to avoid delay in treatment and complications of osteomyelitis and tendon rupture.

Compliance with ethical standards All procedures performed in studies involving human participants were in accordance with the ethical standards of the institutional and/or national research committee and with the 1964 Helsinki declaration and its later amendments or comparable ethical standards.

Grant funding This work received no grant funding.

Informed consent This article does not contain sensitive patient data. The work has been approved by our institutional Office of Research Assurances (ORA) and Institutional Review Board (IRB).

Conflict of interest The authors have no conflict of interest.

\section{References}

1. Pattamapaspong N, Muttarak M, Sivasomboon C. Tuberculosis arthritis and tenosynovitis. Semin Musculoskelet Radiol. 2011;15(5): 459-69.

2. Small LN, Ross JJ. Suppurative tenosynovitis and septic bursitis. Infect Dis Clin N Am. 2005;19(4):991-1005.

3. Aguiar RO, Gasparetto EL, Escuissato DL, et al. Radial and ulnar bursae of the wrist: cadaveric investigation of regional anatomy with ultrasonographic-guided tenography and MR imaging. Skeletal Radiol. 2006;35(11):828-32.

4. Fussey JM, Chin KF, Gogi N, Gella S, Deshmukh SC. An anatomic study of flexor tendon sheaths: a cadaveric study. J Hand Surg Eur Vol. 2009;34(6):762-5.

5. Chau CL, Griffith JF, Chan PT, et al. Rice-body formation in atypical mycobacterial tenosynovitis and bursitis: findings on sonography and MR imaging. AJR Am J Roentgenol. 2003;180(5):1455-9.

6. Hsu CY, Lu HC, Shih TT. Tuberculous infection of the wrist: MRI features. AJR Am J Roentgenol. 2004;183(3):623-8.

7. Lee EY, Rubin DA, Brown DM. Recurrent Mycobacterium marinum tenosynovitis of the wrist mimicking extraarticular synovial chondromatosis on MR images. Skeletal Radiol. 2004;33(7):405-8.

8. Jbara M, Patnana M, Kazmi F, Beltran J. MR imaging: arthropathies and infectious conditions of the elbow, wrist, and hand. Radiol Clin N Am. 2006;44(4):625-42. 